Pacific Journal of Mathematic 


\title{
SUBSYSTEMS OF THE POLYNOMIAL SYSTEM
}

\author{
Frank OKOH AND Frank A. ZorzitTo
}

A pair of complex vector spaces $(V, W)$ is a system if there is a C-bilinear map from $\mathbf{C}^{2} \times V$ to $W$. Given any $\mathbf{C}[\zeta]$-module $M$, and $(a, b)$ a fixed basis of $\mathbf{C}^{2},(M, M)$ is a system with $a m=m, b m=\zeta m$ for all $m$ in $M$. If $M=\mathrm{C}[\zeta]$, the system $P=(M, M)$ is called the polynomial system. The emphasis here is on the disparateness between the polynomial system and the polynomial module. It is shown that each nonzero formal power series in $\mathrm{C}[[\zeta]]$ determines a rank two subsystem of $P$. Among the consequences of this result are that:

(1) $P$ contains $c(c=$ cardinality of $C)$ isomorphism classes of indecomposable subsystems of rank two.

(2) There is a complete set of invariants for decomposable extensions of $(0, \mathbf{C})$ by $P$.

It is also shown that extensions of finite-dimensional subsystems by $P$ are isomorphic to subsystems of $P$. Consequently, $P$ contains purely simple subsystems of arbitrary finite rank. Furthermore, a subsystem of $P$ of finite rank is purely simple if and only if it is indecomposable. Finally the purely simple subsystems of $P$ of rank two are shown to satisfy the ascending chain condition but not the descending chain condition.

Introduction. A pair of complex vector spaces $(V, W)$ is a system if there is a $\mathbf{C}$-bilinear map from $\mathbf{C}^{2} \times V$ to $W$. Any $\mathbf{C}[\zeta]$-module $M(\mathbf{C}[\zeta]$ is the ring of complex polynomials) gives rise to a system $(M, M)$ with $a m=m, b m=\zeta m$ where $(a, b)$ is a fixed basis of $\mathbf{C}^{2}$. The category of systems contains, in this way, subcategories equivalent to the category of $\mathrm{C}[\zeta]$-modules. Probably the most significant difference between the theory of systems and that of modules over a principal ideal domain is the existence of purely simple systems of arbitrary finite rank. This paper is a step in the classification of such systems.

We begin with the simplest case: extensions $(V, W)$ of finite-dimensional torsion-free systems by $P=(\mathbf{C}[\zeta], \mathbf{C}[\zeta])$. A formal power series $l=\sum_{k=0}^{\infty} \alpha_{k} \zeta^{k}$ may be regarded as a linear functional on $\mathbf{C}[\zeta]$, via $l\left(\zeta^{k}\right)=$ $\alpha_{k}$. If $V=\mathbf{C}[\zeta], W=V \oplus \mathbf{C} w, w \neq 0$, we make $(V, W)$ into a system by setting $a \zeta^{k}=\zeta^{k}, b \zeta^{k}=\zeta^{k+1}+\alpha_{k} w$. This system, denoted by $(V, W)_{l}$, is an extension of $(0, \mathbf{C} w)$ by $P$. The rank of $(V, W)$, is 2 , as seen in Theorem 3.1 of [6]. It is shown in Theorem 1.13 that any extension of a finite-dimensional indecomposable torsion-free system by $P$ can be put in the above form. This is then used to show in Theorem 1.14 that any extension 
of a finite-dimensional torsion-free system by $P$ is isomorphic to a subsystem of $P$. The following results on $(V, W)_{l}$ are obtained:

(1) The system $(V, W)_{l}$ is purely simple if and only if $l$ is not the expansion of a rational function (Proposition 2.3).

(2) If $(V, W)_{l_{1}}$ is isomorphic to $(V, W)_{l_{2}}$ by $(\phi, \psi)$ then for some $M$, degree $\phi(f)=$ degree $f$ for all $f$ in $V$ with degree $f \geq M$ (Proposition 3.3).

(3) There exist uncountably many purely simple and nonisomorphic extensions of $(0, \mathbf{C} w)$ by $P$ (Theorem 3.2).

(4) There is a complete set of invariants for decomposable extensions of $(0, C w)$ by $P$, and there are $\boldsymbol{\aleph}_{0}$ isomorphism classes of such extensions (Theorem 3.8).

Now let $X_{l}=\operatorname{ker} l, Y=\mathbf{C}[\zeta]$. Then $\left(X_{l}, Y\right)$ is a subsystem of $(V, W)_{l}$ and a subsystem of $P$. The following results are obtained:

(1) $(V, W)_{l}$ is purely simple if and only if $\left(X_{l}, Y\right)$ is purely simple.

(2) $(V, W)_{l_{1}}$ is isomorphic to $(V, W)_{l_{2}}$ if and only if $\left(X_{l_{1}}, Y\right)$ is isomorphic to $\left(X_{l}, Y\right)$.

(3) Every infinite-dimensional subsystem of $P$ of rank two is isomorphic to $\left(X_{l}, Y\right)$ for some appropriate linear functional $l$ on $\mathrm{C}[\zeta]$. The first two results give in Theorem $3.8(\mathrm{~b})$ that $P$ contains uncountably many isomorphism classes of purely simple subsystems of rank two - a far cry from the structure of $\mathbf{C}[\zeta]$-submodules of $\mathbf{C}[\zeta]$. What's more, Theorem 1.14 can be used to show that, for any positive integer $n, P$ contains a nonterminating descending chain of purely simple subsystems of rank $n$. We do only the case $n=2$.

For all undefined terms on systems we refer to [2] and [6]. $\$ 1$ develops most of the properties of subsystems of $P$ of finite rank needed in $\S \S 2$ and 3 , which contain our main results. We note that the rank one torsion-free system $P$ is denoted on p. 172 of [6] by $P_{\dot{a}}$, where $a \in \mathbf{C}^{2}$, to indicate the dependence of its isomorphism type on the set $\{\alpha a: \in \mathbf{C}\}$. See also p. 285 of [3]. The effect of a change of basis of $\mathbf{C}^{2}$ on $P$ can be deduced from $\mathrm{p}$. 282 of [1].

Finally we remark that any algebraically closed field could be used in place of the complex numbers.

1. Subsystems of $\boldsymbol{P}$ of finite rank. Unless otherwise stated, all systems in this paper are torsion-free. We refer to [2] and [6] for definitions and unexplained notations.

LEMMA 1.1. Let $(V, W)$ be a system. If for any $k$,

$$
\operatorname{tc}_{(V, W)}\left(\phi,\left\{w_{1}, w_{2}, \ldots, w_{k}\right\}\right)
$$


is infinite dimensional, then this subsystem of $(V, W)$ contains an infinite-dimensional pure subsystem of $(V, W)$ of rank not greater than $k$.

Proof. Use induction on $k$. If $k=1$, then $\operatorname{tc}_{(V, W)}\left(\phi,\left\{w_{1}\right\}\right)$ is an infinite-dimensional torsion-closed subsystem of $(V, W)$ of rank 1 . Hence, it is a pure subsystem of $(V, W)$ by Theorem 5.6 of [2]. We assume the result for integers $r, 2 \leq r<k$. Suppose $\operatorname{tc}_{(V, W)}\left(\phi,\left\{w_{1}, w_{2}, \ldots, w_{k}\right\}\right)$ has no direct summand of type $\operatorname{III}^{m}$. Then $\operatorname{tc}_{(V, W)}\left(\phi,\left\{w_{1}, w_{2}, \ldots, w_{k}\right\}\right)$ is already an infinite-dimensional pure subsystem of $(V, W)$ by Theorem 1 of [4]. Also its rank does not exceed $k$. On the other hand, if it has a direct summand of type III $^{m}$, its direct complement is infinite dimensional and of rank not exceeding $k-1$. By the induction hypothesis, that complement contains an infinite-dimensional pure subsystem of $(V, W)$ of rank not exceeding $k-1$.

We now collect some technicalities in 1.2-1.4 which we shall be using constantly. They can all be deduced from results in [2] and [6].

LEMMA 1.2. (a) Let $(V, W)$ be a torsion-free system and $w$ a nonzero element in $W$. The equation $b_{\theta} v=w$ has a solution $v_{\theta}$ in $V$ if and only if $H^{(V, W)}(w)_{\theta}$ is not zero. (For $\boldsymbol{\theta} \in \mathbf{C}, b_{\theta}=b-\theta a$.)

(b) There is a set $\left\{v_{t}\right\}_{l=1}^{n}$ with $b_{\theta} v_{1}=w, a v_{i}=v_{l} ; b_{\theta} v_{1}=v_{1-1} ; 2 \leq i<$ $n+1$, if and only if $H^{(V, W)}(w)_{\theta}=n$ ( $n$ possibly infinite). If $\theta=\infty$, put $a v_{1}=w, b v_{i}=v_{l+1}, 1 \leq i<n+1$.

(c) The sets $\left\{\boldsymbol{v}_{\theta}: \theta \in \tilde{\mathbf{C}}, b_{\theta} v_{\theta}=w\right\}$ and $\left\{v_{l}\right\}_{l=1}^{n}$ are respectively linearly independent.

Lemma 1.3. A subset $S \subset \mathbf{C}[\zeta]$ generates a finite-dimensional subspace of $\mathbf{C}[\zeta]$ if and only if $S$ is of bounded degree, i.e. $\{\operatorname{deg}(f): f \in S\}$ is bounded.

Let $\left(X_{1}, Y_{1}\right) \subset(X, Y) \subset P$ and let $y+Y_{1}$ be a nonzero coset in $Y / Y_{1}$. Suppose $H^{(X, Y) /\left(X_{1}, Y_{1}\right)}\left(y+Y_{1}\right)_{\theta} \neq 0$. Then for some $x$ in $X$,

$$
b_{\theta}\left(x+X_{1}\right)=y+Y_{1}
$$

i.e. $b_{\theta} x-y=y_{1}$, for some $y_{1}$ in $Y_{1}$. Therefore,

$$
x=\left(y+y_{1}\right)(\zeta-\theta)^{-1} .
$$


LEMmA 1.4. If $\left(X_{1}, Y_{1}\right)$ is finite dimensional, in particular if $\left(X_{1}, Y_{1}\right)=$ $(0,0)$, then:

(i) $H^{(X, Y) /\left(X_{1}, Y_{1}\right)}\left(y+Y_{1}\right)_{\theta}=0$ for all but a finite number of $\boldsymbol{\theta}$ in $\tilde{\mathbf{C}}$.

(ii) $H^{(X, Y) /\left(X_{1}, Y_{1}\right)}\left(y+Y_{1}\right)_{\theta}<\infty$ for all $\boldsymbol{\theta}$ in $\mathbf{C}$.

Proof. The set $S=\left\{\left(y+y_{1}\right)(\zeta-\theta)^{-1}: \theta \in \tilde{\mathbf{C}}, y_{1} \in Y_{1}\right\}$ is of bounded degree because $y$ is fixed and $Y_{1}$ is finite-dimensional and hence of bounded degree by 1.3. So by $1.3 \mathrm{~S}$ generates a finite-dimensional subspace of $\mathbf{C}[\zeta]$. Part (i) now follows from 1.2(a) and (c).

(ii) This follows from formula (1) and 1.2(b), 1.2(c), 1.3.

LEMMA 1.5. If $H^{(X, Y) /\left(X_{1}, Y_{1}\right)}\left(y+Y_{1}\right)_{\infty}$ is infinite and $\left(X_{1}, Y_{1}\right)$ is finitedimensional, then $P /(X, Y)$ is finite dimensional.

Proof. If $H^{(X, Y) /\left(X_{1}, Y_{1}\right)}\left(y+Y_{1}\right)_{\infty}=\infty$, then from $1.2(\mathrm{~b})$ and the method used to obtain (1) we deduce that $X$ contains the set

$$
\begin{aligned}
T=\left\{y+y_{1}^{\prime}, \zeta\left(y+y_{1}\right)+y_{2}^{\prime}, \zeta^{2}\left(y+y_{1}\right)+\zeta_{2}^{\prime}+y_{3}^{\prime},\right. \\
\left.\zeta^{3}\left(y+y_{1}\right)+\zeta^{2} y_{2}^{\prime}+\zeta y_{3}^{\prime}+y_{4}^{\prime}, \ldots\right\},
\end{aligned}
$$

where $y_{1}^{\prime} \in Y_{1}$. If $n=$ degree $y$, then $\left\{1, \zeta, \zeta^{2}, \ldots, \zeta^{n-1}, Y_{1}, T\right\}$ spans $\mathbf{C}[\zeta]$, and so $P /(X, Y)$ is finite-dimensional, since $Y_{1}$ is finite-dimensional.

COROllary 1.6. Let $(X, Y)$ be an infinite-dimensional subsystem of $P$ of finite rank. Then $P /(X, Y)$ is finite-dimensional.

Proof. Use induction on rank of $(X, Y)=k$ (say). Let $k=1$, and let $y$ be a nonzero element of $Y$. By Lemma 1.4 with $\left(X_{1}, Y_{1}\right)=(0,0)$, we have $H^{(X, Y)}(y)_{\theta}=0$ for all but a finite number of $\theta \in \tilde{\mathbf{C}}$, and $H^{(X, Y)}(y)_{\theta}$ $<\infty$ for all $\theta$ in $\mathbf{C}$. Since $(X, Y)$ is infinite-dimensional and of rank 1 , $H^{(X, Y)}(y)_{\infty}$ must be infinite by Theorem 3.4 of [2], i.e. $X$ contains $\left\{\zeta^{n} y\right.$ : $n=0,1,2, \ldots\}$. If $m=$ degree $y$, the dimension of $P /(X, Y)$ is not greater than $2 m+1$. We assume the result for all infinite-dimensional subsystems of $P$ of rank not greater than $k-1$. Let $\left(X_{1}, Y_{1}\right)=$ $\operatorname{tc}_{(X, Y)}\left(\phi,\left\{y_{1}, y_{2}, \ldots, y_{k-1}\right\}\right)$ where $\left\{y_{1}, y_{2}, \ldots, y_{k}\right\}$ is a basis of $(X, Y)$ with respect to generation. If $\left(X_{1}, Y_{1}\right)$ is infinite-dimensional we would be done by the induction hypothesis. So we may assume that it is finite-dimensional. Now we note that $(X, Y) /\left(X_{1}, Y_{1}\right)$ is an infinite-dimensional torsion-free system of rank one. By $1.4, H^{(X, Y) /\left(X_{1}, Y_{1}\right)}\left(y+Y_{1}\right)_{\theta}=0$ for all but a finite number of $\theta$ in $\mathbf{C}$, and $H^{(X, Y) /\left(X_{1}, Y_{1}\right)}\left(y+Y_{1}\right)_{\theta}<\infty$ for all $\theta$ in C, provided $y+Y_{1}$ is a nonzero coset. Therefore by Theorem 3.4 of [2], 
$H^{(X, Y) /\left(X_{1}, Y_{1}\right)}\left(y+Y_{1}\right)_{\infty}$ is infinite. So by $1.5, P /(X, Y)$ is finite-dimensional.

Corollary 1.7. Let $\left(X_{1}, Y_{1}\right) \subset(X, Y) \subset P$ where $\left(X_{1}, Y_{1}\right)$ is finite-dimensional and $(X, Y) /\left(X_{1}, Y_{1}\right)$ is infinite-dimensional, torsion-free and of rank one. Then $(X, Y) /\left(X_{1}, Y_{1}\right)$ is isomorphic to $P$.

Proof. This follows from 1.4 and Theorem 3.4 of [2].

In order to avoid circumlocution we shall freely confuse systems and their isomorphism types. Thus we may talk of a system of type III $^{m} \oplus P$ when we mean a system $(V, W)=\left(V_{1}, W_{1}\right) \dot{+}\left(V_{2}, W_{2}\right)$, where $\left(V_{1}, W_{1}\right)$ is of type III $^{m}$ and $\left(V_{2}, W_{2}\right)$ is isomorphic to $P$.

THEOREM 1.8. A subsystem of $P$ of finite rank is indecomposable if and only if it is purely simple. If it is not purely simple, it has a direct summand of type III $^{m}$.

Proof. A purely simple system is necessarily indecomposable. So let $(X, Y) \subset P$ be an indecomposable subsystem of finite rank. Suppose it has a proper pure subsystem $\left(X_{0}, Y_{0}\right)$. By Theorem 5.5 of [1] and the hypothesis on $(X, Y),\left(X_{0}, Y_{0}\right)$ is not finite-dimensional. It is also of finite rank, by Lemma 2.1(a) and Theorem 2.4 of [2]. By 1.6, $P /\left(X_{0}, Y_{0}\right)$ and hence $(X, Y) /\left(X_{0}, Y_{0}\right)$ is finite-dimensional. By the definition of purity this implies that $\left(X_{0}, Y_{0}\right)$ is a direct summand of $(X, Y)$, contradicting the hypothesis that $(X, Y)$ is indecomposable. Therefore $(X, Y)$ has no proper pure subsystems, i.e. it is purely simple. The above also shows that if $(X, Y)$ is not purely simple then it has a finite-dimensional direct summand, and so by Theorem 4.3 of [1], $(X, Y)$ has a direct summand of type III $^{m}$.

COROllary 1.9. An infinite-dimensional subsystem $(X, Y)$ of $P$ of finite rank is of the form

$$
(X, Y)=\left(X_{1}, Y_{1}\right) \dot{+}\left(X_{2}, Y_{2}\right)
$$

where $\left(X_{1}, Y_{1}\right)$ is finite-dimensional and $\left(X_{2}, Y_{2}\right)$ is infinite-dimensional and purely simple. Moreover, the system $\left(X_{2}, Y_{2}\right)$ is unique.

Proof. If $(X, Y)$ is indecomposable then by 1.8 we may take $\left(X_{1}, Y_{1}\right)$ $=0$ and $\left(X_{2}, Y_{2}\right)=(X, Y)$. Otherwise, successive application of 1.8 leads to $(X, Y)=\left(X_{1}, Y_{1}\right)+\left(X_{2}, Y_{2}\right)$, where $\left(X_{1}, Y_{1}\right)$ is of finite rank and a direct sum of subsystems of type III ${ }^{m}$ for various integers $m$, and $\left(X_{2}, Y_{2}\right)$ 
is infinite-dimensional and purely simple. Since $\left(X_{1}, Y_{1}\right)$ is finite-dimensional it remains only to prove the uniqueness of $\left(X_{2}, Y_{2}\right)$. For that we recall that for $y \in Y, \theta \in \tilde{C}$, and $y=y_{1}+y_{2}, y_{i} \in Y_{i}, i=1,2$,

$$
H^{(X, Y)}(y)_{\theta}=\inf \left\{H^{(X, Y)}\left(y_{1}\right)_{\theta}, H^{(X, Y)}\left(y_{2}\right)_{\theta}\right\} .
$$

Suppose $(X, Y)=\left(X_{1}^{\prime}, Y_{1}^{\prime}\right) \dot{+}\left(X_{2}^{\prime}, Y_{2}^{\prime}\right)$ with $\left(X_{1}^{\prime}, Y_{1}^{\prime}\right)$ finite-dimensional and $\left(X_{2}^{\prime}, Y_{2}^{\prime}\right)$ purely simple and infinite-dimensional. Let $M=$ $\max \left\{m:\left(X_{1}^{\prime}, Y_{1}^{\prime}\right)\right.$ or $\left(X_{1}, Y_{1}\right)$ has a direct summand of type III $\left.^{m}\right\}$. Since $\left(X_{2}^{\prime}, Y_{2}^{\prime}\right)$ has no direct summand of type III $^{m}$ for any $m$, every finite-dimensional subsystem of $\left(X_{2}^{\prime}, Y_{2}^{\prime}\right)$ is contained in a subsystem of type $\mathrm{III}^{k_{1}} \oplus \cdots \oplus \mathrm{III}^{k_{t}}$ for some integer $t$ with $\min \left\{k_{1}, \ldots, k_{t}\right\}>M$, by Theorem 2 of [4]. From this and (2) we deduce that $\left(X_{2}^{\prime}, Y_{2}^{\prime}\right) \subset\left(X_{2}, Y_{2}\right)$. Similarly $\left(X_{2}, Y_{2}\right) \subset\left(X_{2}^{\prime}, Y_{2}^{\prime}\right)$. Hence $\left(X_{2}, Y_{2}\right)=\left(X_{2}^{\prime}, Y_{2}^{\prime}\right)$.

Corollary 1.10. An infinite-dimensional subsystem $(X, Y)$ of $P$ of rank two that is not purely simple is of type III $^{m} \oplus P$ for an appropriate integer $m$.

Proof. The hypothesis and 1.9 imply that $(X, Y)=\left(X_{1}, Y_{1}\right) \dot{+}$ $\left(X_{2}, Y_{2}\right)$, where $\left(X_{1}, Y_{1}\right)$ is finite-dimensional and of rank 1, hence of type III ${ }^{m}$ by Theorem 2.2 of [2], and $\left(X_{2}, Y_{2}\right)$ is infinite-dimensional of rank 1. By 1.4 and Theorem 3.4 of [2], $\left(X_{2}, Y_{2}\right)$ is isomorphic to $P$.

Proposition 1.11. An infinite-dimensional subsystem of $P$ of finite rank is an extension of a finite-dimensional system by a system isomorphic to $P$.

Proof. Let $(X, Y) \subset P$ be infinite-dimensional and of finite rank. By 1.9, $(X, Y)=\left(X_{1}, Y_{1}\right) \dot{+}\left(X_{2}, Y_{2}\right)$, where $\left(X_{1}, Y_{1}\right)$ is finite-dimensional and $\left(X_{2}, Y_{2}\right)$ is purely simple and infinite-dimensional. If $\operatorname{rank}\left(X_{2}, Y_{2}\right)$ is 1, then $\left(X_{2}, Y_{2}\right)$ is of type $P$ by 1.4 and Theorem 3.4 of [2]. In that case $(X, Y)$ is trivially an extension of a finite-dimensional system by $P$. Suppose then that $\operatorname{rank}\left(X_{2}, Y_{2}\right)=r>1$. Let $\left\{y_{1}, y_{2}, \ldots, y_{r-1}\right\}$ be part of a basis of $\left(X_{2}, Y_{2}\right)$ with respect to generation. By Lemma 1.1, $\left(X_{3}, Y_{3}\right)=$ $\operatorname{tc}_{\left(X_{2}, Y_{2}\right)}\left(\phi,\left\{y_{1}, y_{2}, \ldots, y_{r-1}\right\}\right)$ must be finite-dimensional because $\left(X_{2}, Y_{2}\right)$ is purely simple. By $1.7,\left(X_{2}, Y_{2}\right) /\left(X_{3}, Y_{3}\right)$ is isomorphic to $P$. Hence $(X, Y)$ is an extension of the finite-dimensional system $\left(X_{1}, Y_{1}\right)+\left(X_{3}, Y_{3}\right)$ by a system isomorphic to $P$.

We want to prove the converse to Proposition 1.11. 
Lemma 1.12. An extension ( $V, W)$ of a finite-dimensional torsion-free system $\left(V_{1}, W_{1}\right)$, by a system $\left(V_{2}, W_{2}\right)$, isomorphic to $P$ is isomorphic to a subsystem of an extension of a system of type III $^{1}$ by $P$.

Proof. Let $\left(V_{1}, W_{1}\right)$ be of type $\mathrm{III}^{k_{1}} \oplus \mathrm{III}^{k_{2}} \oplus \cdots \oplus \mathrm{III}^{k_{t}}$ (say). Let $M=t\left(k_{1}+k_{2}+\cdots+k_{t}\right)$. By using chain representations of systems of type III ${ }^{m}$, we see that $\left(V_{1}, W_{1}\right)$ can be embedded in a system $\left(V_{3}, W_{3}\right)$ of type III ${ }^{M}$. The extension of $\left(V_{1}, W_{1}\right)$ by $\left(V_{2}, W_{2}\right)$ gives the diagram below by pushout:

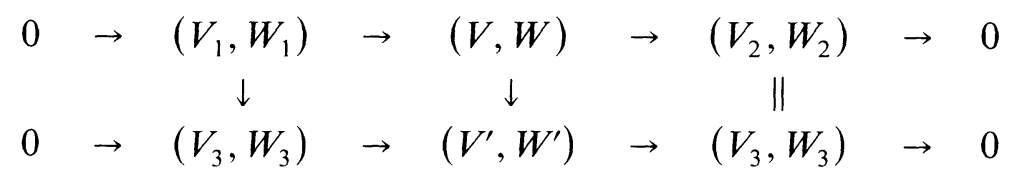

Thus $(V, W)$ is embedded in $\left(V^{\prime}, W^{\prime}\right)$. By Lemma 1.11 of $[6],\left(V^{\prime}, W^{\prime}\right)$ is also an extension of a system of type III $^{1}$ by $P$.

Given the vector spaces $V=\mathbf{C}[\zeta], W=\mathbf{C}[\zeta] \oplus[w]$, with $w \neq 0$, a fixed basis $(a, b)$ of $\mathbf{C}^{2}$ and a linear functional $l: \mathbf{C}[\zeta] \rightarrow \mathbf{C}$, the system defined by the action

$$
a \zeta^{k}=\zeta^{k}, \quad b \zeta^{k}=\zeta^{k+1}+\alpha_{k} w,
$$

where $k=0,1,2, \ldots$ and $\alpha_{k}=l\left(\zeta^{k}\right)$, shall be denoted by $(V, W)_{l}$.

THEOREM 1.13. Every extension of a system of type $\mathrm{III}^{m}$ by $P$ is isomorphic to some $(V, W)$.

Proof. By Lemma 1.11 of [6], such an extension is isomorphic to a system $(V, W)$ where $V=\mathbf{C}[\zeta]$ and $W=\mathbf{C}[\zeta] \oplus[w], w \neq 0$. By Theorem 5.3 of [7] it follows that $(V, W)$ is isomorphic to $(V, W)$, for some functional $l$.

THEOREM 1.14. Every extension of a finite-dimensional torsion-free system by $P$ is isomorphic to a subsystem of $P$.

Proof. By 1.12 and 1.13 it is enough to embed the system $(V, W)$, into $P$. Given $(V, W)$, let $\alpha_{k}=l\left(\zeta^{k}\right)$ for $k=0,1,2, \ldots$, and let $p_{0}, p_{1}, p_{2}, \ldots$ be the polynomials recursively defined by $p_{0}=\zeta, p_{n+1}=\zeta p_{n}-\alpha_{n}$. The mapping $(\phi, \psi):(V, W)_{l} \rightarrow P$, defined by $\psi(w)=1, \phi\left(\zeta^{k}\right)=\psi\left(\zeta^{k}\right)=p_{k}$ for $k=0,1,2, \ldots$, provides a suitable system homomorphism. Indeed $\phi$ and $\psi$ are monomorphisms because the $p_{n}$ 's are linearly independent. Also for the base $(a, b)$ in $\mathbf{C}^{2}$ acting in $(V, W)_{l}$ and in $P$ we have 
and

$$
\psi\left(a \zeta^{k}\right)=\psi\left(\zeta^{k}\right)=\phi\left(\zeta^{k}\right)=a \phi\left(\zeta^{k}\right)
$$

$$
\psi\left(b \zeta^{k}\right)=\psi\left(\zeta^{k+1}+\alpha_{k} w\right)=p_{k+1}+\alpha_{k} 1=\zeta p_{k}=\zeta \phi\left(\zeta^{k}\right)=b \phi\left(\zeta^{k}\right),
$$

for $k=0,1,2, \ldots$

COROllary 1.15. Every extension of a system of type III $^{m}$ by $P$ is isomorphic to a subsystem $(X, Y)$ of $P$ where $X$ is of codimension one in $\mathrm{C}[\zeta]$ and $Y$ is $\mathbf{C}[\zeta]$.

Proof. Such an extension is isomorphic to some $(V, W)$, by 1.13 ; and the embedding $(\phi, \psi):(V, W) \rightarrow P$ of 1.14 is such that $X=\phi(V)$ is of codimension one in $\mathbf{C}[\zeta]$ and $Y=\psi(W)$ is $\mathbf{C}[\zeta]$.

2. Construction of purely simple subsystems of $\boldsymbol{P}$. We shall make no distinction between the formal power series $l=\sum_{k=0}^{\infty} \alpha_{k} \xi^{k} \in \mathbf{C}[[\zeta]]$ and the linear functional on $\mathbf{C}[\zeta]$ it determines. As in the introduction and $\S 1$, the rank two system constructed from $l$ will be denoted by $(V, W)_{l}$. If $f(\zeta)=a_{0}+a_{1} \zeta+\cdots+a_{n} \zeta^{n}, a_{0} \neq 0, \tilde{f}(\zeta)$ will denote the polynomial $a_{0} \zeta^{n}+a_{1} \zeta^{n-1}+\cdots+a_{n}$. Since $\tilde{f}(\zeta)$ is obtained from $f(\zeta)$ by dividing $f(\zeta)$ by $\zeta^{n}$ and replacing $1 / \zeta$ by $\zeta$, this operation preserves divisibility. That is, $g h=f$ if and only if $\tilde{g} \tilde{h}=\tilde{f}$.

Proposition 2.1. Let $l=\sum_{k=0}^{\infty} \alpha_{k} \zeta^{k}$ be a power series expansion of $f(\zeta)=p(\zeta) / q(\zeta)$ where $p(\zeta)=p_{0}+p_{1} \zeta+\cdots+p_{n} \zeta^{n}, q(\zeta)=q_{0}+q_{1} \zeta$ $+\cdots+q_{m} \zeta^{m}$, with $p_{n}, q_{0}, q_{m}$ not zero and $p(\zeta), q(\zeta)$ relatively prime. Then ker $l$ contains the ideal generated by $r(\zeta)=\zeta^{t} q(\zeta), t=\max (0, n-m+1)$. Furthermore ker l contains no larger ideal.

Proof. Assume $n \geq m$. By equating coefficients in $l \cdot q(\zeta)=p(\zeta)$ we get:

$$
\begin{gathered}
\alpha_{0} q_{0}=p_{0} \\
\alpha_{1} q_{0}+\alpha_{0} q_{1}=p_{1} \\
\vdots \\
\alpha_{m} q_{0}+\alpha_{m-1} q_{1}+\cdots+\alpha_{0} q_{m}=p_{m}
\end{gathered}
$$

$$
\begin{gathered}
\alpha_{n} q_{0}+\alpha_{n-1} q_{1}+\cdots+\alpha_{n-m} q_{m}=p_{n} \neq 0 \\
\vdots \\
\alpha_{n+k} q_{0}+\alpha_{n+k-1} q_{1}+\cdots+\alpha_{n+k-m} q_{m}=0 \text { for } k=1,2, \ldots
\end{gathered}
$$


Equation (3) implies that $l\left(\zeta^{k-1} r(\zeta)\right)=0$ for all $k=1,2, \ldots$, where $r(\zeta)=\zeta^{n-m+1} q(\zeta)$. Hence the ideal generated by $r(\zeta)$ is in $\operatorname{Ker} l$. Now suppose Ker $l$ contains the ideal generated by a polynomial $s(\zeta)$ and $s(\zeta)$ divides $r(\zeta)$ Let $s(\zeta)=s_{j}+s_{j-1} \zeta+\cdots+s_{0} \zeta^{j}$, with $s_{0} \neq 0$. We have $l\left(\zeta^{h} s(\zeta)\right)=0$ for $k=0,1,2, \ldots$ by assumption. This means that

$$
s_{0} \alpha_{j+k}+s_{1} \alpha_{j+k-1}+\cdots+s_{j} \alpha_{k}=0 \text { for } k=0,1,2, \ldots
$$

Since $f(\zeta)$ has $\sum_{k=0}^{\infty} \alpha_{k} \zeta^{k}$ as its power series expansion, we may recover $f(\zeta)$ from (4) in the classical fashion (see for instance p. 392 of [5]) as follows:

$$
\begin{gathered}
s_{0} f(\zeta)=s_{0} \alpha_{0}+s_{0} \alpha_{1} \zeta+s_{0} \alpha_{2} \zeta^{2}+\cdots+s_{0} \alpha_{\jmath} \zeta^{j}+\cdots \\
s_{1} \zeta f(\zeta)=s_{1} \alpha_{0} \zeta+s_{1} \alpha_{1} \zeta^{2}+\cdots+s_{1} \alpha_{J-1} \zeta^{\jmath}+\cdots+s_{1} \alpha_{J+k-1} \zeta^{j+k}+\cdots,
\end{gathered}
$$

$$
s_{j} \zeta^{j} f(\zeta)=s_{j} \alpha_{0} \zeta^{\prime}+\cdots+s_{j} \alpha_{k} \zeta^{j+k}+\cdots .
$$

Add the above equations to get $\left(s_{0}+s_{1} \zeta+\cdots+s_{j} \zeta^{\prime}\right) f(\zeta)=t(\zeta)$, where $t(\zeta)$ is a polynomial. Indeed, for $k=0,1,2, \ldots$, the $\zeta^{j+k}$ terms on the right-hand side cancel because of (4). Therefore we get $p(\zeta) / q(\zeta)=$ $t(\zeta) / \tilde{s}(\zeta)$. Since $p(\zeta)$ and $q(\zeta)$ are relatively prime we deduce that $q(\zeta)$ divides $\tilde{s}(\zeta)$, hence $\tilde{q}(\zeta)$ divides $s(\zeta)$. But we had supposed that $s(\zeta)$ divided $\zeta^{n-m+1} \tilde{q}(\zeta)$. This implies that $s(\zeta)=\zeta^{n} \tilde{q}(\zeta)$, where $u \leq n-m+$ 1. If we had $u<n-m+1$, then $l\left(\zeta^{-1} r(\zeta)\right)=\alpha_{n} q_{0}+\alpha_{n-1} q_{1}$ $+\cdots+\alpha_{n-m} q_{m}=0$. This is a contradiction because $p_{n} \neq 0$. Therefore $s(\zeta)=\zeta^{n-m+1} \tilde{q}(\zeta)=r(\zeta)$. If $n<m$, we proceed as above. To obtain equations (3) in that case, $r(\zeta)=q(\zeta)$ works.

A byproduct of the proof of Proposition 2.1 is the following result.

COROLlaRy 2.2. Let $l=\sum_{k=0}^{\infty} \alpha_{k} \zeta^{k} \in F[[\zeta]], F$ any field. Then $l$ is the formal power series expansion of a rational function if and only if the following equivalent conditions are satisfied:

(a) For some positive integers $m, n$, there exist $q_{0}, q_{1}, \ldots, q_{m}$ in $F$ not all zero such that equation (3) is satisfied.

(b) Ker $l$ contains a nonzero ideal of $F[\zeta]$ generated by

$$
\left(q_{0}+q_{1} \zeta+\cdots+q_{m} \zeta^{m}\right) \zeta^{n} .
$$

We remark that (b) is merely a restatement of (a), and (a) is well known (see p. 392 of [5]). 
We shall now show that $P$ and $(V, W)$, share a common subsystem, $\left(X_{l}, Y\right)$, that reflects important properties of $(V, W)_{l}$. Let

$$
X_{l}=\operatorname{Ker} l \subset \mathbf{C}[\zeta], \quad Y=\mathbf{C}[\zeta] .
$$

The system $\left(X_{l}, Y\right)$, with $a x=x, b x=\zeta x$ for all $x \in X_{l}$, is a subsystem of $P$ and also a subsystem of $(V, W)_{l}$. If $l \neq 0,\left(X_{l}, Y\right)$ is a proper subsystem of $P$. We note that $\left(X_{l}, Y\right)$ is not isomorphic to the system $(X, Y)$ of Corollary 1.15 , even though we do not pursue the matter further here.

Proposition 2.3. The system $(V, W)$, is not purely simple if and only if the following equivalent conditions are satisfied:

(i) Statement (a) of Corollary 2.2

(ii) Statement (b) of Corollary 2.2

(iii) $X_{l}$ contains a nonzero ideal.

Proof. The conditions are clearly equivalent. Suppose $(V, W)$, is not purely simple. Then by 1.14 and 1.10 it contains a subsystem isomorphic to $P$. This implies, using the system operation in $(V, W)_{l}$, that $\operatorname{Ker} l$ contains a nonzero ideal. Therefore, $\left(X_{l}, Y\right)$ contains a subsystem isomorphic to $P$. Conversely, if $\operatorname{Ker} l$ contains a nonzero ideal $\langle p(\zeta)\rangle$, then $\operatorname{tc}_{(V, W)_{l}}(\varnothing,\{p(\zeta)\})$ would be infinite-dimensional of rank 1; and by Lemma 1.1 , the rank two system $(V, W)$, would not be purely simple.

From now on we shall assume that all our linear functionals are nonzero and all ideals are nonzero $\mathbf{C}[\zeta]$-ideals. We want to prove that $\operatorname{rank}\left(X_{l}, Y\right)$ is 2 .

Lemma 2.4. If $(X, Y)$ is a subsystem of $P$ and $X$ is of codimension $n$ in $Y$, then $(X, Y)$ does not have a torsion-closed subsystem of type III $^{m_{1}} \oplus$ III $^{m_{2}} \oplus \cdots \oplus$ III $^{m_{n+1}}$.

Proof. Suppose $\left(X_{1}, Y_{1}\right)$ is a torsion-closed subsystem of $(X, Y)$ of type III $^{m_{1}} \oplus$ III $^{m_{2}} \oplus \cdots \oplus$ III $^{m_{n+1}}$. Then there exist linearly independent elements $y_{1}, y_{2}, \ldots, y_{n+1}$ in $Y_{1}$ such that $X_{1} \cap\left[y_{1}, y_{2}, \ldots, y_{n+1}\right]=0$. Since $X$ is of codimension $n$ in $Y$, there exist complex numbers $c_{1}, c_{2}, \ldots, c_{n+1}$ not all zero such that $y=\sum_{l=1}^{n+1} c_{l} y_{l}$ is in $X$. Since $a y=y$, this implies that $(X, Y) /\left(X_{1}, Y_{1}\right)$ has the image of $y$ in $X / X_{1}$ as an eigenvector, contradicting the hypothesis that $\left(X_{1}, Y_{1}\right)$ is torsion-closed in $(X, Y)$.

Lemma 2.5. (a) The system $\left(X_{l}, Y\right)$ has no direct summand of type III $^{m_{1}} \oplus$ III $^{m_{2}}$. (b) If $X_{l}$ contains no ideal then $(X, Y)$ has no direct summand of type III $^{m}$. 
Proof. Since $X_{l}$ is of codimension 1 in $\mathbf{C}[\zeta], 2.5(\mathrm{a})$ follows from 2.4.

For the proof of (b), suppose $\left(X_{l}, Y\right)=\left(X_{1}, Y_{1}\right) \dot{+}\left(X_{2}, Y_{2}\right)$ with $\left(X_{1}, Y_{1}\right)$ of type III $^{m}$. Then $\operatorname{dim}\left(X_{l} / X_{2}\right)=m-1$ and $\operatorname{dim}\left(Y / Y_{2}\right)=m$. Since $X_{l}$ is of codimension 1 in $Y$ this implies $\operatorname{dim}\left(Y / X_{2}\right)=m$. Since $a X_{2}=X_{2} \subset Y_{2}$, that implies $X_{2}=Y_{2}$. In particular, $\zeta X_{2} \subset X_{2}$, contradicting the hypothesis that $X_{l}$ does not contain an ideal.

Lemma 2.6. If $(X, Y)$ is a subsystem of $P$ and $X$ is of codimension $n$ in $Y$, then $(X, Y)$ contains an infinite-dimensional pure subsystem of rank not exceeding $n+1$.

Proof. If $\operatorname{rank}(X, Y)$ is less than or equal to $n+1$ there is nothing to prove. So we may suppose that $\operatorname{rank}(X, Y) \geq n+2$. Let $\left\{y_{1}, y_{2}, \ldots, y_{n+1}\right\}$ be part of a basis of $(X, Y)$ with respect to generation. Let $\left(X_{1}, Y_{1}\right)=$ $\operatorname{tc}_{(X, Y)}\left(\varnothing,\left\{y_{1}, y_{2}, \ldots, y_{n+1}\right\}\right)$. If $\left(X_{1}, Y_{1}\right)$ is finite-dimensional then by Theorem 4.3 of $[1]$ and the fact that $\operatorname{rank}\left(X_{1}, Y_{1}\right)=n+1,\left(X_{1}, Y_{1}\right)$ is of type $\mathrm{III}^{m_{1}}+\ldots+\mathrm{III}^{m_{n+1}}$, contradicting 2.4 . Therefore $\left(X_{1}, Y_{1}\right)$ is infinite-dimensional and an appeal to 1.1 gives us the required result.

THEOREM 2.7. If $(X, Y)$ is a subsystem of $P$ and $X$ is of codimenson one in $Y$, then the rank of $(X, Y)$ is two. In particular, the rank of $\left(X_{l}, Y\right)$, where $Y=\mathbf{C}[\zeta]$, is two.

Proof. Suppose $X$ contains an ideal $\langle p(\zeta)\rangle$. Then

$$
\left(X_{1}, Y_{1}\right)=t c_{(X, Y)}(\varnothing,\{p(\zeta)\})
$$

is an infinite-dimensional subsystem of $P$ of rank 1. By 1.6, $P /\left(X_{1}, Y_{1}\right)$, hence $\left(X_{1}, Y_{1}\right) /\left(X_{1}, Y_{1}\right)$ is finite-dimensional. By 1.1, $\left(X_{1}, Y_{1}\right)$ is pure in $(X, Y)$. By the definition of purity, $\left(X_{1}, Y_{1}\right)$ is a direct summand of $(X, Y)$ with a finite-dimensional complement $\left(X_{2}, Y_{2}\right)$ (say). By Theorem 4.3 of [1], $\left(X_{2}, Y_{2}\right)$ is a direct sum of subsystems of type III $^{m}$. By 2.5(a) there can only be one such direct summand. That is, $\left(X_{2}, Y_{2}\right)$ is of type III $^{m}$. Therefore, $\operatorname{rank}\left(X_{2}, Y_{2}\right)=\operatorname{rank}\left(X_{1}, Y_{1}\right)=1$. Thus $\operatorname{rank}(X, Y)=2$.

Suppose $X$ does not contain an ideal. If $\operatorname{rank}(X, Y) \geq 3$ then $(X, Y)$ contains an infinite-dimensional pure subsystem $\left(X_{1}, Y_{1}\right)$ of rank $\leq 2$, by 2.6, since $X$ is of codimension 1 in $\mathrm{C}[\zeta]$. By 1.6, $P /\left(X_{1}, Y_{1}\right)$ and $(X, Y) /\left(X_{1}, Y_{1}\right)$ are finite-dimensional. Therefore $(X, Y)$ contains a direct summand of type III $^{m}$, contradicting Lemma 2.6(b). So $\operatorname{rank}(X, Y) \leq 2$. If $\operatorname{rank}(X, Y)=1$, then from 1.4 and Theorem 3.4 of [2] $(X, Y)$ is isomorphic to $P$. This means $X$ would contain a nonzero ideal. Therefore $\operatorname{rank}(X, Y)$ is 2 . 
Proposition 2.8. The system $(V, W)$, is purely simple if and only if $\left(X_{l}, Y\right)$ is purely simple.

Proof. Suppose $(V, W)_{l}$ is not purely simple. Then by $1.14,1.10$ and 2.3 in that order, $\left(X_{l}, Y\right)$ contains a subsystem isomorphic to $P$. The torsion-closure in $\left(X_{l}, Y\right)$ of such a subsystem is a rank 1 infinite-dimensional subsystem of the rank two system $\left(X_{l}, Y\right)$. Hence by $1.1,\left(X_{l}, Y\right)$ is not purely simple. Conversely, if $\left(X_{l}, Y\right)$ is not purely simple, 1.10 and 2.3 yield that $(V, W)_{l}$ is not purely simple.

The next result shows that $\operatorname{Ker} l$ captures the essence of $(V, W)_{l}$.

THEOREM 2.9. If $l_{1}, l_{2}$ are in $\mathrm{C}[[\zeta]]$ then $(V, W)_{l_{1}}$ is isomorphic to $(V, W)_{l_{2}}$ if and only if $\left(X_{l_{1}}, Y\right)$ is isomorphic to $\left(X_{l_{2}}, Y\right)$.

Proof. Suppose $(\phi, \psi):(V, W)_{l_{1}} \rightarrow(V, W)_{l_{2}}$ is an isomorphism. Since $e \phi(f)=\psi(e f)$ for all $e \in \mathbf{C}^{2}$ and $f$ in $V$, we conclude from the respective system operations that $\phi\left(X_{l_{1}}\right)=X_{l_{2}}$ and $\psi(Y)=Y$. Therefore $(\phi, \psi)$ restricted to $\left(X_{l_{1}}, Y\right)$ is an isomorphism onto $\left(X_{l_{2}}, Y\right)$.

For the converse, we first note the following. Let $l \in \mathbf{C}[[\zeta]]$. By 1.11 and 2.7 and Theorems 2.4 and 2.2 of [2], we have the exact sequence

$$
0 \rightarrow\left(X_{1}, Y_{1}\right) \rightarrow\left(X_{l}, Y\right) \rightarrow P \rightarrow 0,
$$

where $\left(X_{1}, Y_{1}\right)$ is of type III $^{m}$. Let $v \in V \backslash X$. We have $a v=v \in Y$. Since $v \notin X_{l}, b v=\zeta v+\beta w$ for some $\beta \neq 0$. So in $(V, W)_{l} /\left(X_{l}, Y\right), a v=0$ and $b v \neq 0$. Therefore $(V, W)_{l} /\left(X_{l}, Y\right)$ is of type $\mathrm{II}_{\infty}^{1}$. From (5) we obtain the long exact sequence:

$$
\operatorname{Hom}\left(\mathrm{II}_{\infty}^{1}, P\right) \rightarrow \operatorname{Ext}\left(\mathrm{II}_{\infty}^{1}, \mathrm{III}^{m}\right) \rightarrow \operatorname{Ext}\left(\mathrm{II}_{\infty}^{1},\left(X_{l}, Y\right)\right) \rightarrow \operatorname{Ext}\left(\mathrm{II}_{\infty}^{1}, P\right) .
$$

The first entry is 0 because $P$ has no eigenvalues. From the table in [3], we cull the following: $\operatorname{dim} \operatorname{Ext}\left(\mathrm{II}_{\infty}^{1}, \mathrm{III}^{m}\right)=1$ and $\operatorname{dim} \operatorname{Ext}\left(\mathrm{II}_{\infty}^{1}, P\right)=0$. Hence $\operatorname{Ext}\left(\mathrm{II}_{\infty}^{1},\left(X_{l}, Y\right)\right)$ is also one-dimensional. Namely, all nonsplit extensions are isomorphic.

Let $(\phi, \psi):\left(X_{l_{1}}, Y\right) \rightarrow\left(X_{l_{2}}, Y\right)$ be an isomorphism. A pushout and the fact that $(V, W)_{l_{1}} /\left(X_{l_{1}}, Y\right)$ is of type $\mathrm{II}_{\infty}^{1}$ yield the following commutative diagram with exact rows:

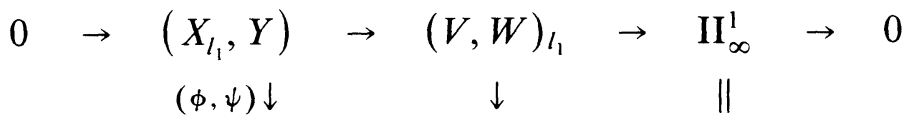

$$
\begin{aligned}
& 0 \rightarrow\left(X_{l_{2}}, Y\right) \rightarrow(U, Z) \rightarrow \mathrm{II}_{\infty}^{1} \quad \rightarrow \quad 0
\end{aligned}
$$


Therefore $(V, W)_{l_{1}}$ is isomorphic to $(U, Z)$. But $(V, W)_{l_{2}}$ is also a nonsplit extension of $\left(X_{l_{2}}, Y\right)$ by $\mathrm{II}_{\infty}^{1}$. It is nonsplit because it is torsion-free, while $\mathrm{II}_{\infty}^{1}$ has $\infty$ as an eigenvalue. Therefore $(U, Z)$ is isomorphic to $(V, W)_{l_{2}}$, and hence $(V, W)_{l_{1}}$ is isomorphic to $(V, W)_{l_{2}}$.

Proposition 2.10. Every infinite-dimensional subsystem $\left(X^{\prime}, Y^{\prime}\right)$ of $P$ of rank two is isomorphic to $\left(X_{l}, Y\right)$ for an appropriate linear functional $l$ on

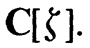

Proof. By 1.11, $\left(X^{\prime}, Y^{\prime}\right)$ is an extension of a finite-dimensional system $\left(X_{1}, Y_{1}\right)$ by a system isomorphic to $P$. Since $\operatorname{rank}\left(X^{\prime}, Y^{\prime}\right)=2$ and $\operatorname{rank} P$ $=1, \operatorname{rank}\left(X_{1}, Y_{1}\right)=1$ by Theorem 2.4 of [2]. Therefore $\left(X_{1}, Y_{1}\right)$ is of type III $^{m}$. By 1.11 of [6], $\left(X^{\prime}, Y^{\prime}\right)$ is also an extension of a system of type III $^{1}$ by $P$. Hence it is isomorphic to a subsystem $(X, Y)$ of $P$ with $X$ of codimension one in $\mathbf{C}[\zeta]$ and $Y=\mathbf{C}[\zeta]$ by 1.15 . Therefore $X$ is the kernel $X_{l}$ of a linear functional $l$ on $\mathbf{C}[\zeta]$ and $\left(X^{\prime}, Y^{\prime}\right)$ is isomorphic to $\left(X_{l}, Y\right)$.

COROllaRy 2.11. If $\beta$ is a nonzero complex number, $l_{1}$ a linear functional on $C[\zeta]$ and $l_{2}=\beta l_{1}$. Then $(V, W)_{l_{1}}$ is isomorphic to $(V, W)_{l_{2}}$.

Proof. This is immediate from 2.9 because $\operatorname{Ker} l_{1}=\operatorname{Ker} l_{2}$. So $\left(X_{l_{1}}, Y\right)$ $=\left(X_{l_{2}}, Y\right)$.

3. Some invariants. We begin the section with a description of a complete set of invariants for completely decomposable subsystems of $P$ of rank two.

Proposition 3.1. The system $\left(X_{l}, Y\right)$ has the form $\left(X_{l}, Y\right)=\left(X_{1}, Y_{1}\right)$ $\dot{+}\left(X_{2}, Y_{2}\right)$ with $\left(X_{1}, Y_{1}\right)$ of type $\mathrm{III}^{n}$ and $\left(X_{2}, Y_{2}\right)=(p(\zeta) \cdot \mathbf{C}[\zeta], p(\zeta) \cdot$ $\mathrm{C}[\zeta])$ with degree $p(\zeta)=n$, if and only if $\langle p(\zeta)\rangle$ is the largest ideal contained in $X_{l}$.

Proof. Suppose $\left(X_{l}, Y\right)=\left(X_{1}, Y_{1}\right)+\left(X_{2}, Y_{2}\right)$ with $\left(X_{1}, Y_{1}\right)$ of type III $^{n}$ and $\left(X_{2}, Y_{2}\right)=(p(\zeta) \cdot \mathbf{C}[\zeta], p(\zeta) \cdot \mathbf{C}[\zeta])$, where $\operatorname{deg} p(\zeta)=n$. Clearly the ideal $\langle p(\zeta)\rangle$ is in $X_{l}$. If $X_{l}$ contains an ideal $\langle q(\zeta)\rangle$, then $\left(X_{l}, Y\right)$ contains the rank one infinite-dimensional subsystem, $\left(X_{3}, Y_{3}\right)=$ $\operatorname{tc}_{\left(X_{l}, Y\right)}(\varnothing,\{q(\zeta)\})$. The latter is isomorphic to $P$. By 1.1 and 2.7, $\left(X_{3}, Y_{3}\right)$ is a proper pure subsystem of $\left(X_{l}, Y\right)$. By 1.6, $P /\left(X_{3}, Y_{3}\right)$, hence $\left(X_{l}, Y\right) /\left(X_{3}, Y_{3}\right)$, is finite-dimensional. This makes $\left(X_{3}, Y_{3}\right)$ a direct summand of $\left(X_{l}, Y\right)$ isomorphic to $P$ with a finite-dimensional direct complement. By $1.9,\left(X_{3}, Y_{3}\right)=\left(X_{2}, Y_{2}\right)$. Thus $\langle q(\zeta)\rangle \subseteq\langle p(\zeta)\rangle$. 
Conversely, suppose $\langle p(\zeta)\rangle$ is the largest ideal in $X_{l}$. By 2.3, 2.7, and $1.10,\left(X_{l}, Y\right)$ is of type III ${ }^{m} \oplus P$. Let $\left(X_{l}, Y\right)=\left(X_{3}, Y_{3}\right)+\left(X_{4}, Y_{4}\right)$ with $\left(X_{3}, Y_{3}\right)$ of type III ${ }^{m}$ and $\left(X_{4}, Y_{4}\right)$ isomorphic to $P$. In particular, $\left(X_{4}, Y_{4}\right)$ $=(q(\zeta) \cdot \mathbf{C}[\zeta], q(\zeta) \cdot \mathbf{C}[\zeta])$ for some polynomial $q(\zeta)$. So $X_{l}$ contains the ideal $\langle q(\zeta)\rangle$. Therefore $\langle q(\zeta)\rangle \subseteq\langle p(\zeta)\rangle$. Hence $\left(X_{4}, Y_{4}\right) \subseteq(p(\zeta)$. $\mathbf{C}[\zeta], p(\zeta) \cdot \mathbf{C}[\zeta])$. The argument in the last paragraph gives $(p(\zeta)$. $\mathbf{C}[\zeta], p(\zeta) \cdot \mathbf{C}[\zeta]) \subseteq\left(X_{4}, Y_{4}\right)$. Therefore $\left(X_{4}, Y_{4}\right)=(p(\zeta) \cdot \mathbf{C}[\zeta], p(\zeta)$. C $[\zeta])$. If $n=$ degree $p(\zeta)$, then $\operatorname{dim} X_{3}=\operatorname{dim} Y_{3}-1=n-1$ so $m=n$, as required.

An equivalence relation on rational functions of the form $p(\zeta) / q(\zeta)$, where $\zeta$ does not divide $q(\zeta)$, is defined by

$$
p_{1}(\zeta) / q_{1}(\zeta) \equiv p_{2}(\zeta) / q_{2}(\zeta)
$$

if $m_{1}+\max \left(0, n_{1}-m_{1}+1\right)=m_{2}+\max \left(0, n_{2}-m_{2}+1\right)$, where $n_{i}=$ degree $p_{\imath}(\zeta), m_{l}=$ degree $q_{i}(\zeta), i=1,2$. Let $D$ be the resulting set of equivalence classes. From 2.1,3.1, 2.9, and 2.10 we obtain the following classification theorem.

THEOREM 3.2. The set $D$ is a complete set of invariants for the isomorphism classes of decomposable extensions of III $^{1}$ by $P$ and decomposable infinite-dimensional subsystems of $P$ of rank two, respectively. Furthermore there are only countably many such classes.

We now turn our attention to purely simple subsystems of $P$ of rank two. The next proposition provides an entering wedge.

Proposition 3.3. If $(\phi, \psi)$ is an isomorphism from $(V, W)_{l_{1}}$ onto $(V, W)_{l_{2}}$, then there exists a positive integer $M$ such that $\operatorname{deg} p(\zeta)=$ $\operatorname{deg} \phi(p(\zeta))$, whenever $p(\zeta)$ is a polynomial in $V$ of degree not less than $M$.

Proof. Let $(a, b)$ be the fixed basis of $\mathbf{C}^{2}$ used to define the given systems. Then $\phi\left(\zeta^{n}\right)=a \phi\left(\zeta^{n}\right)=\psi\left(a \zeta^{n}\right)=\psi\left(\zeta^{n}\right)$ for $n=0,1,2, \ldots$ Let $p_{n}$ be this common polynomial. In the range space of $(V, W)_{l_{1}}, \zeta^{k}=$ $\zeta^{k}+\alpha_{k-1} w-\alpha_{k-1} w$ where $\alpha_{k}=l_{1}\left(\zeta^{k}\right)$. So $\psi\left(\zeta^{k}\right)=\psi\left(\zeta^{k}+\alpha_{k-1} w\right)-$ $\alpha_{k-1} \psi(w)$. That is,

$$
p_{k}=\psi\left(b \zeta^{k-1}\right)-\alpha_{k-1} \psi(w)=b \phi\left(\zeta^{k-1}\right)-\alpha_{k-1} \psi(w) .
$$


Since $p_{k}$ is a polynomial, the $w$-component of $b \phi\left(\xi^{k-1}\right)$ is equal to the $w$-component of $\alpha_{k-1} \psi(w)$. Denoting this component by $\psi(w)_{p}$ we get from (6)

$$
p_{k}=\zeta p_{k-1}-\alpha_{k-1} \psi(w)_{p} .
$$

Also (6) gives the following recursive relation for $p_{k}$ :

$$
\begin{aligned}
p_{k}= & \zeta_{p_{0}}^{k}-\alpha_{0} \zeta^{k-1} \psi(w)_{p}-\alpha_{1} \zeta^{k-2} \psi(w) p-\cdots-\alpha_{k-2} \zeta \psi(w)_{p} \\
& -\alpha_{k-1} \psi(w)_{p} .
\end{aligned}
$$

Since $\left[p_{0}, p_{1}, p_{2}, \ldots\right]=\mathbf{C}[\zeta]$ there exists an integer $n$ such that degree $p_{n}$ $>$ degree $\psi(w)_{p}$. Since $p_{n+1}=\zeta p_{n}-\alpha_{n} \psi(w)_{p}$, it follows that degree $p_{n+1}$ $=$ degree $p_{n}+1$. This argument repeated gives degree $p_{n+k}=$ degree $p_{n}+$ $k$ for $k=1,2,3, \ldots$. Since $\phi$ is an isomorphism, the codimension $n$ of $\left[\zeta^{n}, \zeta^{n+1}, \ldots\right]$ in the domain space of $(V, W)_{l_{1}}$ equals that of its image $\left[p_{n}, p_{n+1}, \ldots\right]$ in the domain space of $(V, W)_{l_{2}}$. Therefore degree $p_{n}=n$ and so degree $p_{n+k}=n+k$ for $k=0,1,2, \ldots$ Let $m=$ $\max \left\{\right.$ degree $\left.p_{j}: j=1, \ldots, n-1\right\}$. The required $M$ of the proposition is any integer greater than $m+n$.

Let $F$ be the field $\mathbf{Z} / 2 \mathbf{Z}$ and choose a set $S$ of representatives for a basis of the $F$-vector space $\Pi_{\aleph_{0}} F / \oplus_{\aleph_{0}} F$. The set $S$ has the following properties:

(i) $\operatorname{Card} S=2^{\aleph_{0}}$.

(ii) For $S=\left(s_{j}\right)_{j=0}^{\infty}$ in $S$ the set $\left\{j \in \mathbf{N}: s_{j}=1\right\}$ is finite.

(iii) For two distinct elements $s, t$ in $S$ the set $\left\{j \in \mathbf{N}: s_{J} \neq t_{j}\right\}$ is infinite.

For any positive integer $r$ put $f(r)=\sum_{i=1}^{r-1} i !+r$, and $f(0)=0$. We note that for $r \geq 4$,

$$
r !>f(r)
$$

For each $s=\left(s_{J}\right)_{j=0}^{\infty}$ in $S$ consider the sequence $l_{s}$, whose $n$th term is $s_{r}$ if $n=f(r)$ for some $r$ and is 0 if $n \neq f(r)$ for any $r$. The set $T$ of such $l_{s}$ 's is uncountable. The elements of $T$ are simply sequences of the form $\left(0 s_{1} 0 s_{2} 00 s_{3} 000000 s_{4} 00 \ldots\right)$, where $\left(s_{j}\right)_{j=0}^{\infty} \in S$ and the number of 0 's between successive $s_{j}$ 's is 1 !, 2!, 3!, etc. Any sequence $l$ from $T$ is to be identified with a formal power series and hence a linear functional on $\mathrm{C}[\zeta]$ in the natural manner. From 2.2 any $l \in T$ cannot be the expansion of a rational function. For each $l \in T$ the system $(V, W)$, is therefore purely simple, by 2.3. Our goal is to prove that the different $(V, W)$,'s are not isomorphic. 
LEMmA 3.4. If $l=\left(\alpha_{k}\right)_{k=0}^{\infty}$ is in $T$ and for some $k \geq 8, \alpha_{k-1}=1$ and $\alpha_{k}=0$, then $H^{(V, W)_{1}}\left(\zeta^{j}\right)_{\infty}<H^{(V, W)_{1}}\left(\zeta^{k}\right)_{\infty}$ for all $j=0,1,2, \ldots, k-1$.

Proof. Since $\alpha_{k-1}=1, k-1=f\left(r_{0}\right)$ for some integer $r_{0}$. Since $k-1$ $\geq 7, r_{0} \geq 4$. For $0 \leq j \leq k-1, H^{(V, W)_{1}}\left(\zeta^{j}\right)_{\infty} \leq f\left(r_{0}\right)$ while $H^{(V, W)_{1}}\left(\zeta^{k}\right) \geq$ $r_{0}$ !. The result then follows from (7).

Let $l_{l}=\left(\alpha_{k l}\right)_{k=0}^{\infty} i=1,2$, be two elements in $T$. Suppose $(\phi, \psi)$ : $(V, W)_{l_{1}} \rightarrow(V, W)_{l_{2}}$ is an isomorphism. Let $M$ be an integer such that if degree $f(\zeta)>M$ then degree $\phi(f(\zeta))=$ degree $f$, according to Proposition 3.3.

Lemma 3.5. Suppose $8 \leq M<k$ and $\alpha_{k-1,2}=1, \alpha_{k 2}=0$. Then $\phi\left(\zeta^{k}\right)$ $=c_{k} \zeta^{k}$ for some nonzero complex number $c_{k}$.

Proof. From $\alpha_{k-1,2}=1$ we deduce that $f\left(r_{0}\right)=k-1$ for some integer $r_{0}$. Since $k-1 \geq 7, r_{0} \geq 4$. Also $k \neq f(r)$ for any integer $r$. So $\alpha_{k 1}=0$. Moreover $\alpha_{k+j, 1}=0$ for $0 \leq j \leq r_{0}$ !, by the description of elements in $T$. Therefore $H^{(V, W)_{1}}\left(\zeta^{k}\right)_{\infty} \geq r_{0}$ !. Since an isomorphism of systems preserves height functions, $H^{(V, W)_{l_{2}}}\left(\phi\left(\zeta^{k}\right)\right)_{\infty} \geq r_{0}$ ! By the choice of $k$, degree $\phi\left(\zeta^{k}\right)=k$, say $\phi\left(\zeta^{k}\right)=c_{0}+c_{1} \zeta+\cdots+c_{k} \zeta^{k}$. Since $\alpha_{k-1,2}=$ 1 and $\alpha_{k, 2}=0$, we get from Lemma 3.4 that

$$
H^{(V, W) l_{2}}\left(c_{k} \zeta^{k}\right)_{\infty}>H^{(V, W)_{2}}\left(c_{l} \zeta^{l}\right)_{\infty}
$$

if $0 \leq i<k$ and $c_{i} \neq 0$. Also $H^{(V, W)_{l_{2}}}\left(c_{l} \zeta^{i}\right)_{\infty} \leq f\left(r_{0}\right)$ for such $c_{l}$. Now we recall that if $H^{(V, W)}\left(w_{1}\right)_{\theta} \neq H^{(V, W)}\left(w_{2}\right)_{\theta}$ in a system $(V, W)$, then

$$
H^{(V, W)}\left(w_{1}+w_{2}\right)_{\theta}=\inf \left\{H^{(V, W)}\left(w_{1}\right)_{\theta}, H^{(V, W)}\left(w_{2}\right)_{\theta}\right\}
$$

for any $\theta \in \tilde{\mathbf{C}}$. Since $r_{0} \geq 4, f\left(r_{0}\right)<r_{0}$ ! by (7). Therefore $c_{t}=0$ for $0 \leq i<k$, hence proving the lemma.

REMARK 3.6. Since $l_{1} \neq l_{2}$, they differ in infinitely many spots. So for any integer, in particular for $k>M \geq 8$, there exists a larger integer $t$ such that:

(i) $\alpha_{k-1,2}=1 ; \alpha_{k 2}=0\left(\right.$ so $\left.\alpha_{k 1}=0\right)$.

(ii) $\alpha_{t 1} \neq \alpha_{t 2}$ (one of them is 0 and the other 1).

(iii) for all $j, k \leq j<t, \alpha_{j 1}=\alpha_{j 2}=0$.

Proposition 3.7. If $l_{1}, l_{2}$ are distinct elements of $T$, then $(V, W)_{l_{1}}$ is not isomorphic to $(V, W)_{l_{2}}$. 
Proof. We shall use the notation in Lemma 3.5. Choose $t, k$ with the properties described in 3.6, so that from those properties

$$
H^{(V, W)_{\iota_{1}}}\left(\zeta^{k}\right)_{\infty} \neq H^{(V, W)_{l_{2}}}\left(\beta \zeta^{k}\right)_{\infty}
$$

for any nonzero complex number $\beta$. From Lemma 3.5, we deduce that $(V, W)_{l_{1}}$ is not isomorphic to $(V, W)_{l_{2}}$, because an isomorphism preserves height functions.

In what follows $c=$ cardinality of $\mathbf{C}$.

THEOREM 3.8. (a) There are exactly $c$ isomorphism classes of purely simple extensions of a system of type $\mathrm{III}^{1}$ by $P$.

(b) There are exactly c isomorphism classes of purely simple subsystems of $P$ of rank two.

Proof. By Theorem 1.13 the number of isomorphism classes of extensions of a system of type III $^{1}$ by $P$ is no greater than Card $C[[\zeta]]=c$. But $\operatorname{Card}(T)=c$. The theorem follows from Propositions 3.7 and 1.15.

Lemma 3.9. A purely simple system of rank greater than one is infinitedimensional.

Proof. Let $(V, W)$ be a finite-dimensional torsion-free system. By Theorem 4.3 of $[1],(V, W)$ has a direct summand of type III $^{m}$. Since a system of type III $^{m}$ is of rank $1,(V, W)$ is purely simple if and only if it is of rank 1 .

Proposition 3.10. The system $P$ contains a nonterminating descending chain of purely simple subsystems of rank 2.

Proof. For any $l_{0} \in T$ the system $(V, W)_{l_{0}}$ is purely simple. Let ( $\left.X_{0}, Y_{0}\right)$ be a subsystem $P$ isomorphic to $(V, W)_{l_{0}}$, as in Theorem 1.14. We now show that every purely simple subsystem of $P$ of rank 2 contains a proper purely simple subsystem $\left(X_{k+1}, Y_{k+1}\right)$ also of rank 2. By Lemma $3.9\left(X_{k}, Y_{k}\right)$ is infinite-dimensional. Therefore by Proposition 1.11 it is isomorphic to an extension of a finite-dimensional system by $P$. Since $\left(X_{k}, Y_{k}\right)$ and $P$ are of respective ranks 2 and 1 , the finite-dimensional system is of rank 1 . Therefore $\left(X_{k}, Y_{k}\right)$ is an extension of a system of type III $^{m}$ by a system isomorphic to $P$. So by Theorem 1.13 there is an isomorphism $(\phi, \psi):(V, W)_{l} \rightarrow\left(X_{k}, Y_{k}\right)$ for some $(V, W)_{l}$. By Proposition 2.8 and Theorem $2.7,\left(X_{l}, Y\right)$ is a proper purely simple subsystem of 
$(V, W)_{l}$ of rank 2. So $(\phi, \psi)\left(X_{l}, Y\right)$ is a proper purely simple subsystem of $\left(X_{k}, Y_{k}\right)$ of rank 2. Put $\left(X_{k+1}, Y_{k+1}\right)=(\phi, \psi)\left(X_{l}, Y\right)$. The required nonterminating descending chain of purely simple subsystems of $P$ of rank 2 is $\left(X_{0}, Y_{0}\right) \supset\left(X_{1}, Y_{1}\right) \supset\left(X_{2}, Y_{2}\right) \supset \cdots$.

Proposition 3.11. Any ascending chain of purely simple subsystems of $P$ of finite rank greater than one terminates.

Proof. Let $\left(X_{1}, Y_{1}\right) \subset\left(X_{2}, Y_{2}\right) \subset \cdots$ be an ascending chain of purely simple subsystems of $P$ where $\operatorname{rank}\left(X_{k}, Y_{k}\right) \geq 2$ for $k=1,2, \ldots$ By Lemma 3.9, $\left(X_{k}, Y_{k}\right)$ is infinite-dimensional. By Corollary 1.6, $P /\left(X_{k}, Y_{k}\right)$ is finite-dimensional for all $k=1,2, \ldots$. Therefore the sequence terminates because $\operatorname{dim} P /\left(X_{k}, Y_{k}\right) \geq \operatorname{dim} P /\left(X_{k+1}, Y_{k+1}\right), k=1,2, \ldots$

Using a chain representation for $P$ as on p. 283 of [3], we see that $P$ contains a nonterminating ascending chain of purely simple subsystems: $\left(X_{1}, Y_{1}\right) \subset\left(X_{2}, Y_{2}\right) \subset \cdots \subset\left(X_{n}, Y_{n}\right) \subset \cdots$ where $\left(X_{n}, Y_{n}\right)$ is of type III $^{n}$, and hence of rank one.

REMARK. The set $T$ of Lemma 3.4 can also be used to prove the following results valid for any field $k$.

(1) The rank of $k[[\zeta]]$ as a module over $k[\zeta]$ is $c$.

(2) Let $L$ be the set of $k$-rational functions $p(\zeta) / q(\zeta)$ with $q(0) \neq 0$. Then the dimension of $k[[\zeta]] / L$ as a $k$-vector space is $c$.

\section{REFERENCES}

[1] N. Aronszajn, and U. Fixman, Algebraic spectral problems, Studia Math., 30 (1968), 273-338.

[2] U. Fixman, On algebraic equivalence between pairs of linear transformations, Trans. Amer. Math. Soc., 113 (1964), 424-453.

[3] U. Fixman, and F. Okoh, Extensions of pairs of linear transformations between infinite-dimensional vector spaces, Linear Algebra Appl., 19 (1978), 275-291.

[4] U. Fixman, and F. Zorzitto, A purity criterion for pairs of linear transformations, Canad. J. Math., 26 (1973), 734-745.

[5] G. H. Hardy, A Course in Pure Mathematics, 9th Edition, Cambridge University Press, (1948), Cambridge, England.

[6] F. Okoh, A bound on the rank of purely simple systems, Trans. Amer. Math. Soc., 232 (1977), 169-186.

[7] Torsion-free modules over a noncommutative hereditary ring, Ph.D. Thesis, Queen's University, Kingston, Ontario, Canada (1975). 
Received February 15, 1980. Research by the first author was partially supported by York University Faculty of Arts Research Grant and by the National Sciences and Engineering Research Council of Canada. The second author's research was supported by the National Sciences and Engineering Research Council of Canada.

YORK UNIVERSITY

DOWNSVIEW, ONTARIO

CANADA M3J 1 P3

AND

UNIVERSITY OF WATERLOO

WATERLOO, ONTARIO

CANADA N2L $3 \mathrm{G} 1$ 



\title{
PACIFIC JOURNAL OF MATHEMATICS EDITORS
}

\author{
Donald BabBitT (Managing Editor) \\ University of California \\ Los Angeles, CA 90024 \\ Hugo RossI \\ University of Utah \\ Salt Lake City, UT 84112 \\ C. C. Moore and Arthur Ogus \\ University of California \\ Berkeley, CA 94720
}

J. DugundiI

Department of Mathematics

University of Southern California

Los Angeles, CA 90089-1113

R. FinN and H. SAMELSON

Stanford University

Stanford, CA 94305

\section{ASSOCIATE EDITORS}
R. ARENS
E. F. BECKENBACH
B. H. NeUmanN
F. WOLF
K. YosHida
(1906-1982)

\section{SUPPORTING INSTITUTIONS}

\author{
UNIVERSITY OF ARIZONA \\ UNIVERSITY OF BRITISH COLUMBIA \\ CALIFORNIA INSTITUTE OF TECHNOLOGY \\ UNIVERSITY OF CALIFORNIA \\ MONTANA STATE UNIVERSITY \\ UNIVERSITY OF NEVADA, RENO \\ NEW MEXICO STATE UNIVERSITY \\ OREGON STATE UNIVERSITY
}

\author{
UNIVERSITY OF OREGON \\ UNIVERSITY OF SOUTHERN CALIFORNIA \\ STANFORD UNIVERSITY \\ UNIVERSITY OF HAWAII \\ UNIVERSITY OF TOKYO \\ UNIVERSITY OF UTAH \\ WASHINGTON STATE UNIVERSITY \\ UNIVERSITY OF WASHINGTON
}

The Supporting Institutions listed above contribute to the cost of publication of this Journal, but they are not owners or publishers and have no responsibility for its content or policies.

Mathematical papers intended for publication in the Pacific Journal of Mathematics should be in typed form or offset-reproduced (not dittoed), double spaced with large margins. Please do not use built up fractions in the text of the manuscript. However, you may use them in the displayed equations. Underline Greek letters in red, German in green, and script in blue. The first paragraph must be capable of being used separately as a synopsis of the entire paper. In particular it should contain no bibliographic references. Please propose a heading for the odd numbered pages of less than 35 characters. Manuscripts, in triplicate, may be sent to any one of the editors. Please classify according to the scheme of Math. Reviews, Index to Vol. 39. Supply name and address of author to whom proofs should be sent. All other communications should be addressed to the managing editor, or Elaine Barth, University of California, Los Angeles, California 90024.

There are page-charges associated with articles appearing in the Pacific Journal of Mathematics. These charges are expected to be paid by the author's University, Government Agency or Company. If the author or authors do not have access to such Institutional support these charges are waived. Single authors will receive 50 free reprints; joint authors will receive a total of 100 free reprints. Additional copies may be obtained at cost in multiples of 50 .

The Pacific Journal of Mathematics is issued monthly as of January 1966. Regular subscription rate: $\$ 132.00$ a year (6 Vol., 12 issues). Special rate: $\$ 66.00$ a year to individual members of supporting institutions.

Subscriptions, orders for numbers issued in the last three calendar years, and changes of address should be sent to Pacific Journal of Mathematics, P.O. Box 969, Carmel Valley, CA 93924, U.S.A. Old back numbers obtainable from Kraus Periodicals Co., Route 100, Millwood, NY 10546.

The Pacific Journal of Mathematics ISSN $0030-8730$ is published monthly by the Pacific Journal of Mathematics at P.O. Box 969, Carmel Valley, CA 93924. Application to mail at Second-class postage rates is pending at Carmel Valley, California, and additional mailing offices. Postmaster: Send address changes to Pacific Journal of Mathematics, P. O. Box 969, Carmel Valley, CA 93924.

PUBLISHED BY PACIFIC JOURNAL OF MATHEMATICS, A NON-PROFIT CORPORATION

Copyright $\odot 1983$ by Pacific Journal of Mathematics 


\section{Pacific Journal of Mathematics}

Vol. 109, No. $2 \quad$ June, 1983

Tibor Bisztriczky, On the singularities of almost-simple plane curves . . . . 257

Peter B. Borwein, On Sylvester's problem and Haar spaces .............. 275

Emilio Bujalance, Cyclic groups of automorphisms of compact nonorientable Klein surfaces without boundary ............... 279

Robert Jay Daverman and John J. Walsh, Acyclic decompositions of

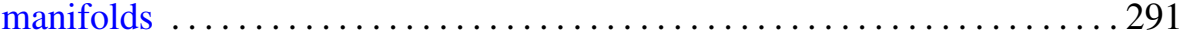

Lester Eli Dubins, Bernstein-like polynomial approximation in higher

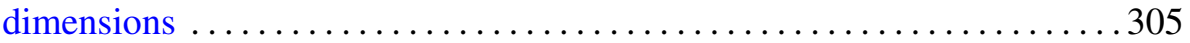

Allan L. Edelson and Jerry Dee Schuur, Nonoscillatory solutions of

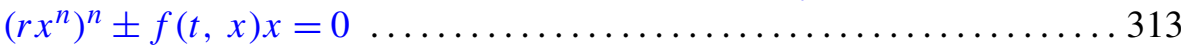

Akira Endô, On units of pure quartic number fields ................. 327

Hector O. Fattorini, A note on fractional derivatives of semigroups and

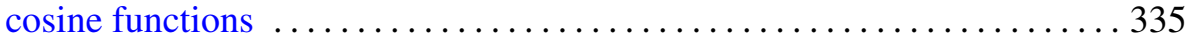

Ronald Fintushel and Peter Sie Pao, Circle actions on homotopy spheres with codimension 4 fixed point set ........................ 349

Stephen Michael Gagola, Jr., Characters vanishing on all but two

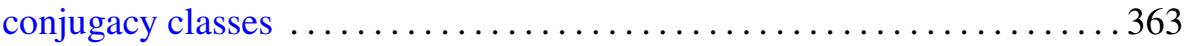

Saverio Giulini, Singular characters and their $L^{p}$ norms on classical Lie

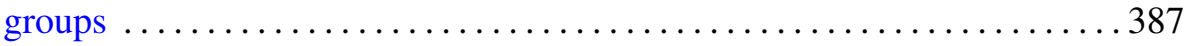

Willy Govaerts, Locally convex spaces of non-Archimedean valued continuous functions

Wu-Chung Hsiang and Bjørn Jahren, A remark on the isotopy classes of diffeomorphisms of lens spaces $\ldots . \ldots \ldots \ldots \ldots \ldots$

Hae Soo Oh, Compact connected Lie groups acting on simply connected 4-manifolds

Frank Okoh and Frank A. Zorzitto, Subsystems of the polynomial system

Knut Øyma, An interpolation theorem for $H_{E}^{\infty}$

Nikolaos S. Papageorgiou, Nonsmooth analysis on partially ordered vector spaces. II. Nonconvex case, Clarke's theory ........ 